\title{
A Sports Teaching Mode Based on Social Networking Service Teaching Resources
}

\author{
https://doi.org/10.3991/ijet.v15i08.13369
}

\author{
Xuelin Yang ${ }^{(凶)}$, Xiaojun Jiang, Li Rong \\ Taizhou Polytechnic College, Taizhou, China \\ 33923367 @qq. com
}

Zhe Xu

Hebei North University, Hebei, China

\begin{abstract}
As today's ideal in regard to health globally goes deep, sports courses receive more and more attention. However, high-quality teaching resource scarcity exists in current PE teaching, thus affecting the teaching effect. In this study, a teaching mode based on Social Networking Service (SNS) teaching resources was designed. Firstly, SNS-based PE teaching network learning community was designed, and self-organized construction of teaching resources was achieved in the learning community, including network courseware, teaching knowledge points, common problems, cases and media materials, etc. Thus, the network teaching mode oriented to self-organization of teaching resources. To test the teaching effect of this new teaching mode, Tai Ji course was used for teaching practice. A kind of virtual software for Tai Ji teaching was used, and virtual 3D technology and multi-functional operating interface were applied to create a brand-new Tai Ji learning mode. The results show that the average score of the students who took the new Tai Ji course teaching mode is much higher than that of students with regular teaching, and the proportion of high score students is also higher, indicating that the teaching mode proposed in this study can significantly improve the teaching effect of Tai Ji course, and also provide a beneficial thought for constructing a new teaching mode for other courses.
\end{abstract}

Keywords-SNS' network teaching; teaching mode; Tai Ji course

\section{Introduction}

As the education system pays more and more attention to teenagers' comprehensive quality, PE course also plays an increasingly important role in the whole course structure. In the higher education system, PE course has become an important part of liberal education and undertakes the role of strengthening students' physique and comprehensive quality. College PE course design is based on relevant courses of PE major, including course content, teaching mode and teacher resource, etc. [1]. As an important constituent part of Chinese traditional martial arts, Tai Ji owns very strong national features and the function of strengthening one's body. 
Carrying out Tai Ji course in colleges can not just let students gain richer methods to do physical exercise, but also promote students' sense of national pride and carry forward Chinese traditional culture. Hence, an increasing number of colleges start to set up the teaching resources about Tai $\mathrm{Ji}$, and good teaching feedback has been obtained [2]. However, current college Tai Ji teaching resources also have certain problems which generate some negative impacts on effective implementation of Tai Ji education. On the one hand, present Tai Ji teaching courses excessive emphasize external teaching and ignore connotation teaching. As a result, many students only learn to imitate the movements of Tai Ji, but fail to grasp the real charm of Tai Ji. Tai $\mathrm{Ji}$ is a Chinese traditional martial art combining the internal and the external, and has high requirements for learners' psychological state and comprehension ability. However, the class hours of college PE teaching are few. Thus, many teachers do not teach theoretical knowledge in teaching, and only teach movements. On the other hand, many colleges lack the teachers with high level, which to certain degree hinders the effective implementation of Tai Ji teaching [3]. As a traditional martial art, Tai Ji is mainly inherited by handing down from the older generations of the family and master- apprentice teaching. Although the movements of Tai Ji have been basically made public, it is hard to really grasp the entire systematical knowledge effectively. Consequently, many students only learn superficial knowledge of Tai Ji, but fail to effectively comprehend its connotation.

SNS refers to Social Networking Service, and its core is sharing, interaction and cooperation among people [4]. Benefiting from the rapid development of internet and intelligent platform, the coverage and influence of SNS improve rapidly. In such case, the teaching method of SNS network teaching resource also has arouses extensive attention and research of education workers. The introduction of SNS into the teaching mainly owns two advantages. On the one hand, with SNS network, the indepth communication and exchange can be conducted between students, between students and teachers, and between teachers, which can play a positive role for students to grasp the connotation of the knowledge learned and for teachers to improve their professional ability [6]. On the other hand, SNS can make high-quality teaching resources shared and transmitted via network. Via SNS network, students can acquire rich high-quality learning resources. Meanwhile, these resources can be updated rapidly to effectively make up for the insufficient learning resources [6]. Seeing from the two points, the teaching method of SNS network teaching resource can actively solve the problems existing in current Tai Ji course. Thus, this study investigated the practice and application of SNS network teaching resource method, in the hope of improving the problems and shortcomings in current course.

\section{State of the Art}

SNS stress the connection of individual-centered social relations. People can share their dynamics and communicate in the form of rich media. Currently, studentcentered teaching has become one of important trends of college course reform. Hence, teaching framework construction based on SNS has aroused the attention of 
many education workers. Lim [7] conducted SNS teaching research by taking South Korean students as the qualitative case study object according to the feature that international students are difficult to adapt to American new life and new culture, including instant message, blog, chatting website and emails of the students. The results show that the use of SNS in different ways contributes to the subjects' emotional health and academic purpose. Hori et al. [8] combined SNS online learning system with open resources of open educational resources (OER) higher education and developed a flexible learning environment based on electronic textbooks. It was found that the learning environment is more beneficial to enhancing students' learning interest. Moreno et al. [9] combined SNS with Facebook and created the learning page and learning team related to open education. The results show that such learning institution can more easily attract students and enhance the learning effect. Zheng et al. [10] applied SNS in the construction of teaching resource sharing platform and let students and teachers jointly participate in the teaching resource construction and management, thus expanding the resource richness. Chen [11] developed a themebased learning design framework based on SNS network. It could be used in the teaching process of many courses focusing on practice, and presented a good application effect on promoting students; problem solving ability and operation ability.

Teacher's teaching ability has a significant effect on course teaching effect. Evaluating teacher's teaching ability in a scientific and rational way plays an important role in promoting teacher resource and teaching effect. At present, teacher's teaching ability evaluation gradually develops to overall reflection of teaching-related abilities, and emphasizes the marching with talent training objective. For example, Huang [12] considered teaching ability assessment of college teachers should be linked to major training objective and course teaching objective, and the specific evaluation indexes and methods were designed from 6 basic elements: knowledge, design, expression, implementation, attitude and effect. Zhao et al. [13] combined actual situations of college PE teaching, and applied Delphi method to dynamically boost and construct teaching ability evaluation index system of college PE teachers containing four indexes (knowledge learning ability, teaching practice ability, teaching research ability and information technology application ability) to truly and accurately embody teacher's teaching ability.

Current defects in college PE teaching are mainly reflected in teacher resource. The insufficient teacher resource leads to the lack of high-quality teaching resources. Meanwhile, the teaching contents fail to really touch subject connotation and essence. For the shortcomings in PE teaching, this paper explored the application mode and practice of SNS network teaching resource method in PE teaching. The innovation points of this study are mainly reflected in two aspects. Firstly, SNS network teaching resource method was applied in Tai Ji course teaching, and the proposed teaching design mode can effectively assisting in solving two main problems existing in current Tai Ji course teaching. Secondly, teacher's professional ability and level have significant influence on effective implementation of Tai Ji course teaching. The deficiency in this aspect is also one of main problems. Teacher ability assessment 
method was proposed on the basis of data mining, and assessment method has positive promotion effect in improving teacher level of Tai Ji course.

\section{Interactive Teaching Mode Based on Educational Games}

\subsection{SNS network}

With the rapid development of internet, network teaching has become a widelyused teaching mode. Especially in the higher education field, it has gained the theoretical research application achievement. Compared with traditional mode, the significant changes have happened to the teaching mode of network teaching system. The relations of each entity are shown in Fig.1. In the network teaching system, network teaching resource is dependent on network teaching environment. Teachers and students release and acquire teaching resources by network learning environment, and they can communicate with each other. The network teaching system has the open characteristic. Teachers can release and update teaching resources anytime, and push them to students in real time. Students can broke through the space-time restriction of traditional teaching mode, conveniently acquire learning resources, independently arrange and allocate learning time.

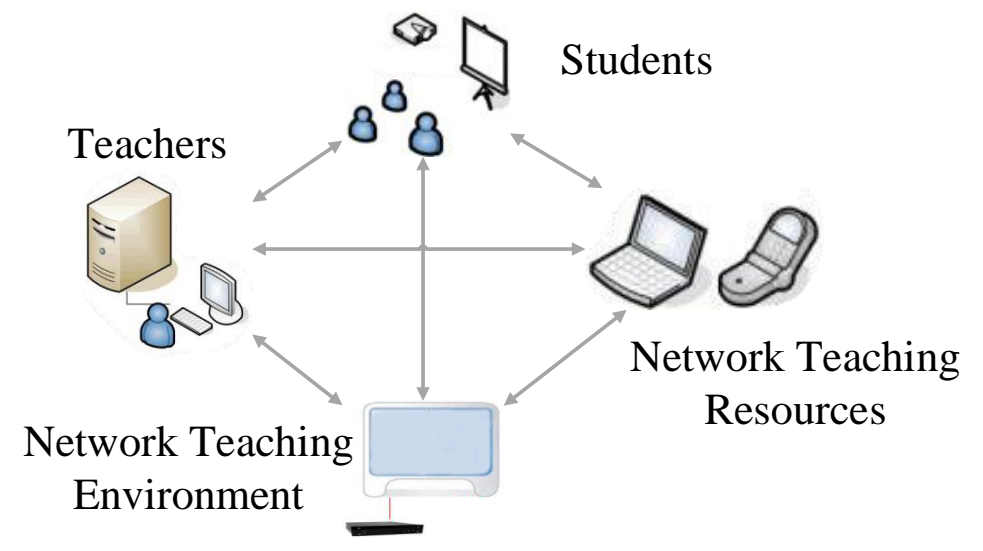

Fig. 1. Network relationship of network teaching system

The full name of SNS is Social Networking Service, and its core is to advocate sharing, interaction and cooperation among people. With SNS platform, people can share and display their personalized contents anytime and anywhere, and can break the time-space limit to interact and cooperate with others, thus creating rich valuable information [12]. Due to this characteristic of SNS, it can be used to construct a learning environment of resource sharing, cooperation and communication for teachers and students. In SNS environment, information spreading mode changes significant, and information acquisition efficiency also improves greatly. As shown in Fig.2 and Fig.3, users passively receive information or gain information sources 
through the search engine. SNS-based information transmission mechanism focuses on active push. The system can actively push the valuable information to users according to their interests, which can have a positive role in promoting information acquisition efficiency.

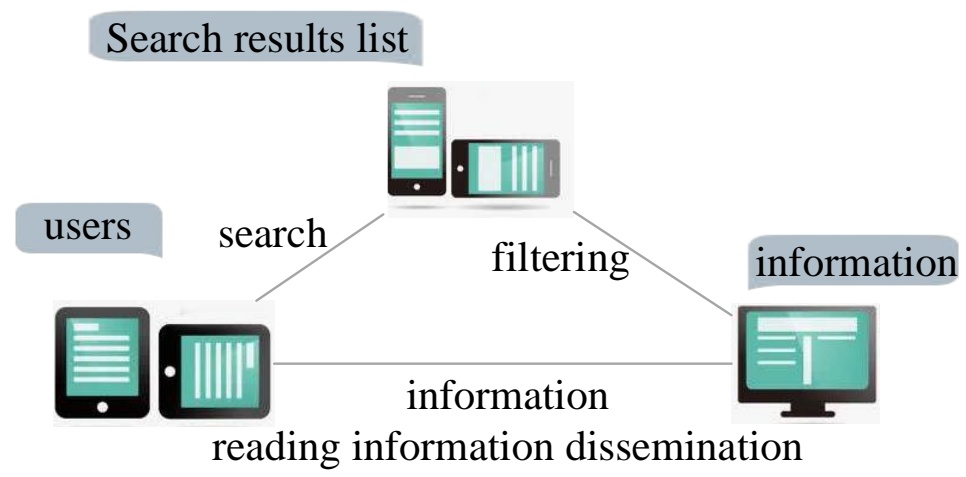

Fig. 2. Traditional information transmission mechanism

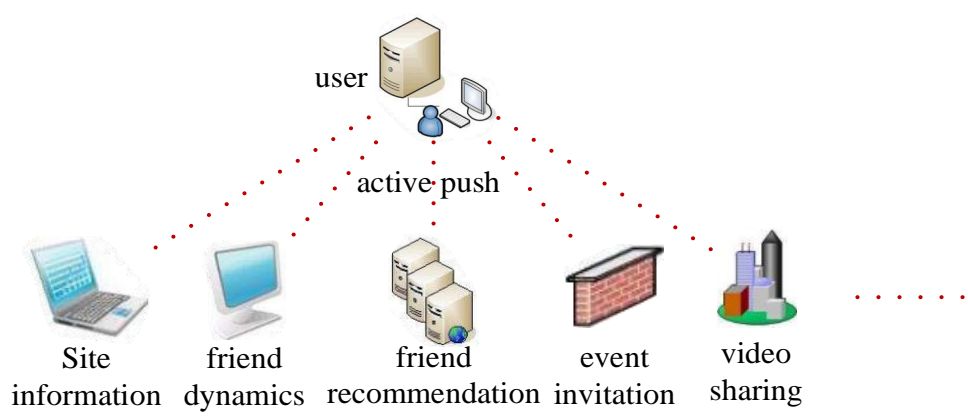

Fig. 3. SNS-based information transmission mechanism

\subsection{Construction method of SNS network teaching resource}

In the self-organization construction model, network teaching environment can form stable teaching resources and environment after the operation for some time, without the need of external intervention or only with the minimum supervision. This study aims to design a SNS-based self-organization construction model of network teaching resources. The teachers and students are both system users and learning resource contributors. The SNS-based self-organization construction model of network teaching resources is shown in Fig.4. In this model, students' communication with teachers and other students and learning resources collected by students will be deemed as the original data of teaching resource and recorded by the system. Teacher's asking and answering will also be used as the important supplement of teaching resources. Through the self-organization construction model of teaching 
resources, a lot of teaching resources come from the experience of students and teachers, and own high value in terms of resource practicability. Students can utilize these resources to improve knowledge mastery depth, and teachers can keep improving their teaching ability.

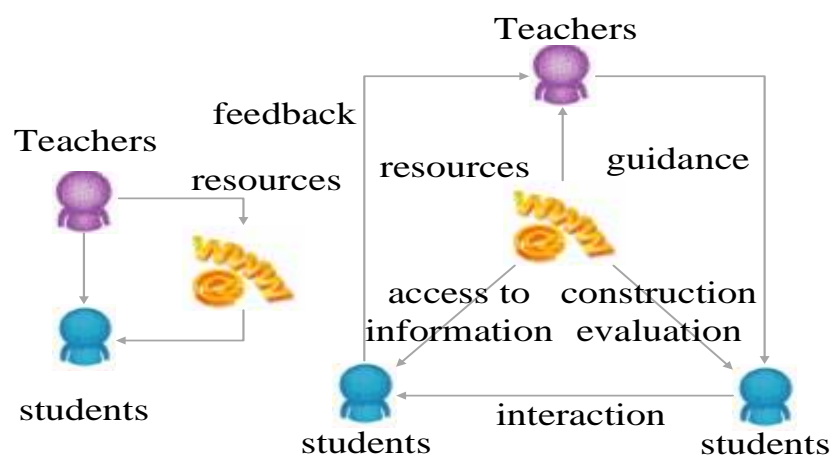

Fig. 4. Self-organization construction model of network teaching resources

\subsection{Network teaching mode oriented to teaching resource self-organization}

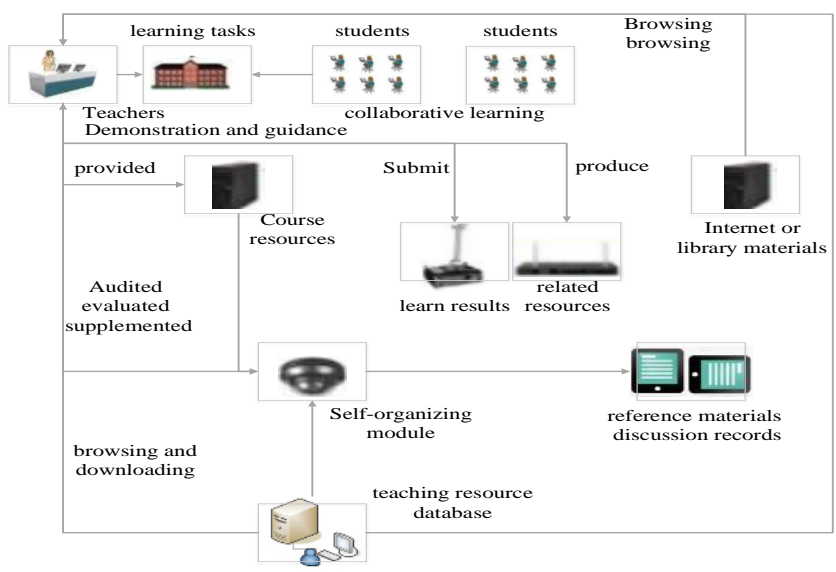

Fig. 5. Network teaching mode oriented to teaching resource self-organization

In SNS-based network teaching, the communication between teachers and students generates original resources. However, these resources cannot be directly used in teaching, and need planning and standardization. In the meantime, after teaching resources form, the continuous interaction and feedback between teachers and students will continuously optimize and improve these resources, thus achieving teaching resource self-organization. The network teaching mode oriented to teaching resource self-organization is shown in Fig.5. The whole process is composed of two parts. The major function of the first part is to achieve the interaction process among 
teachers, students and learning resources. The main function of the second part is to realize resource self-organization process. The cooperation and activity between teachers and students generate original resources. However, these resources cannot be directly stored in the database as the teaching resources. After standardization and normative treatment of these teaching resources by the self-organization module, they can be used as the teaching resources. All proposed teaching resources are stored in the teaching resource library.

\subsection{Design mode of "SNS network teaching resource library" based on PE major}

SNS network teaching resource library is the core of network teaching mode. Storing, updating and optimizing teaching resources need to be achieved by depending on the resource library. According to the SNS-based network teaching mode oriented to teaching resource self-organization, this study designed the system architecture of teaching resource library which is composed of four sub-systems, as shown in Fig.6. Network teaching subsystem realizes teaching and learning functions. Under network support, teaching and learning processes can break through time-space limit and be conducted anytime and anywhere. In resource releasing and browsing subsystem, both teachers and students can become the subject of teaching resources, including actively submitting resources, mutual communication and writing. Resource library management subsystem standardizes and normalizes the original resources contributed by teachers and students to form valuable teaching resources. Resource storage subsystem is used to store all kinds of teaching resources, including network courseware, teaching knowledge points, common problems, cases and media materials.

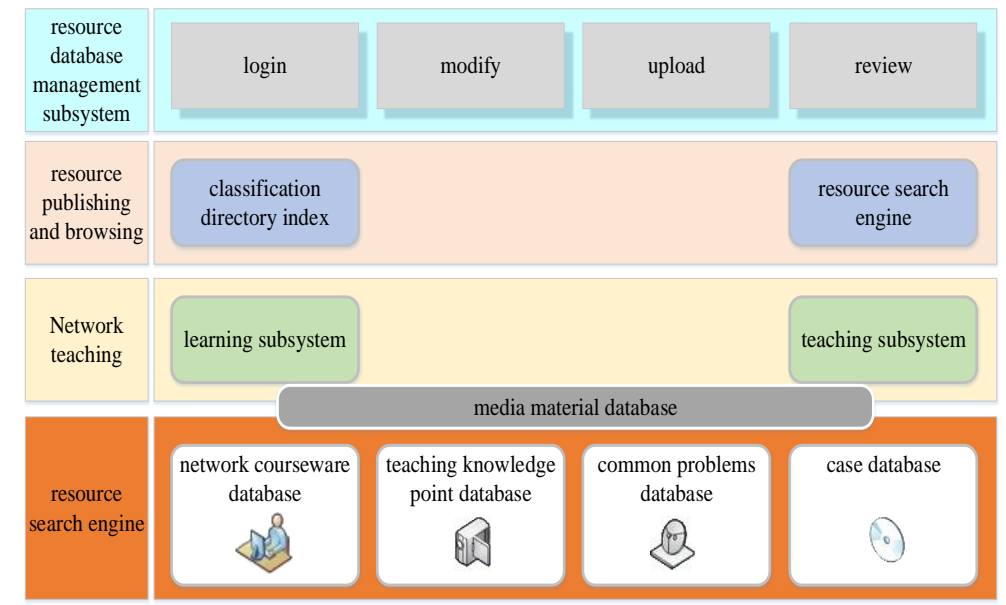

Fig. 6. System architecture of network teaching resource library 
In addition, the network teaching resource library also provides user operating interface for teachers and students. Every user has the sole username and identify. After logging in the resource library, the system will load different function modules according to user identify. The function design of user operating interface is shown in Fig.7, which consists of learning interface, online releasing and backend management modules.

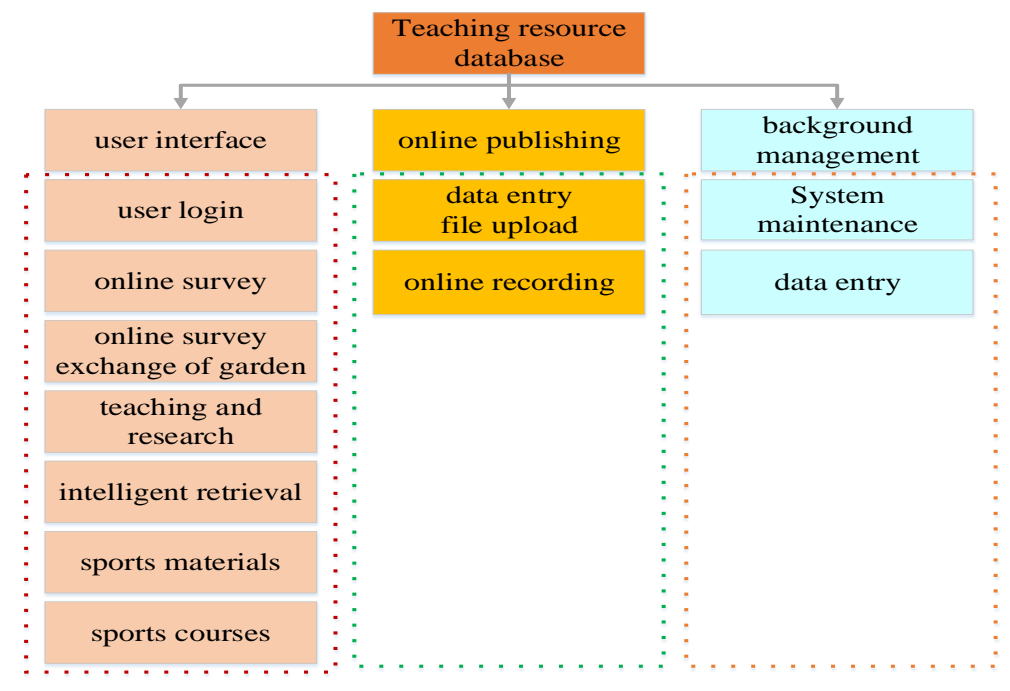

Fig. 7. Function design of user operating interface of network teaching resource library

\subsection{Teacher ability evaluation of SNS network teaching resource}

In the SNS-based network teaching method, learning resources own strong selforganization characteristic. Compared with traditional artificial construction method, the self-organization method does not need manual intervention, and can carry out objective resource library construction under the unified framework. This characteristic can bring the positive influence on real and accurate evaluation of teacher's teaching ability, and can avoid the drawback of manual evacuation, that is the teacher's teaching ability cannot be reliably and truly reflected. In order to achieve this characteristic, a teacher ability evaluation framework based on data mining was constructed in this study, which comprises 6 steps.

\section{Preprocessing of evaluation index database}

Since the data types in the evaluation index database are very numerous and the manifestation modes differ, normative processing is required before data mining. Anonymous coding principle is followed in data preprocessing, and the characteristic variable is discretized according to the pre-established principle. The teacher evaluation data after preprocessing can be expressed with the vector: 


$$
U_{i}=\left[u_{i 1}, u_{i 2}, u_{i 3}, \ldots, u_{i k},\right]
$$

\section{Rough set reduction of teaching data}

To reduce the calculation amount in data mining process, the teaching evaluation data can be reduced by the decision attribute under the precondition of no loss of key information. If the following three conditions are met, the dataset D can be expressed with the reduction set D1 about decision attribute set $\mathrm{E}$, without causing the decision distortion:

$$
\begin{gathered}
D_{1} \subseteq D, D_{1} \neq \varnothing \\
Z_{I_{N D}\left(D_{1}\right)}\left(I_{N D}(E)\right)=Z_{I_{N D}(D)}\left(I_{N D}(E)\right) \\
\text { No } D_{2} \subseteq D_{1},{ }_{\text {Makes }} Z_{I_{N D}\left(D_{2}\right)}\left(I_{N D}(E)\right)=Z_{I_{N D}\left(D_{1}\right)}\left(I_{N D}(E)\right)
\end{gathered}
$$

\section{Association rules mining for teaching data}

After the reduction of the rough set, data mining problem becomes a mining problem of data association rules, i.e. solving the association rules of $D=>E$ in $\mathrm{R}=\{\mathrm{S}, \mathrm{B}, \mathrm{H}, \mathrm{f}\}$.

Input: transaction database D, minimum support threshold min_support;

Output: frequent itemset L in D;

\section{Teaching data mining}

In teaching data mining, decision table $\mathrm{R}$, minimum support $\varepsilon$ and minimum confidence level $\lambda$ need to be input, and association rules mining is implanted.

\section{Principal component analysis of teaching ability}

The purpose of principal component analysis is to determine the weight of each decision index in association rules mining. It utilizes statistical analysis method to process discrete information. Principal component analysis of teaching ability involves 3 steps:

Normalize the data

Judge index correlation and calculate the weight

Gain the composition of principal components an describe them

\section{Evaluation result analysis}

The integration of evaluation results is conducted according to Formula (2). The teachers with higher scores have the stronger teaching ability.

$$
X_{i}=\sum_{j=1}^{q} Q_{j} \cdot P_{i j}
$$




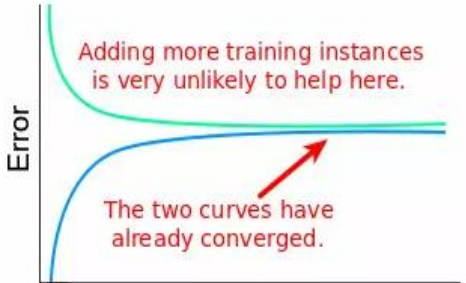

Training set size

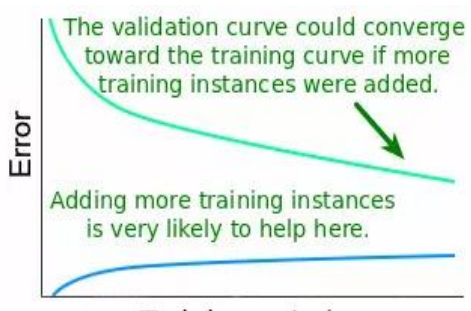

Training set size

Fig. 8. Effect contrast algorithm diagram of two data mining algorithms

It can be seen from the left diagram of Fig.8, when the sample number of the training set increases to a value, the error of verification set basically remains unchanged, indicating that increasing more training data points cannot always bring the better model. It is better to try to construct more complicated model algorithm, instead of increasing the size of training set. The right diagram in Fig. 8 demonstrates that increasing more training samples will lower model error and improve model performance.

\section{$4 \quad$ Teaching Example and Effect}

\subsection{Teaching example}

In this study, the teaching design mode based on SNS network teaching was applied in the teaching practice of Tai Ji course in the sophomore year. In the process of teaching practice, two demands were mainly considered in the design of teaching platform: constructing a convenient cooperation and communication platform for students and teachers, and providing self-organization environment of learning resources. In combination of teaching practice of Tai Ji course in the sophomore year, this study designed teaching resource management and optimization mode, thus effectively solving the problem that there is lack of high-quality Tai Ji teaching resources. Meanwhile, teachers can keep improving their teaching ability through this platform. The teaching resource management and optimization mode of Tai Ji course in the sophomore year is shown in Fig.9. The resource management and optimization basically achieve self-organization treatment. 


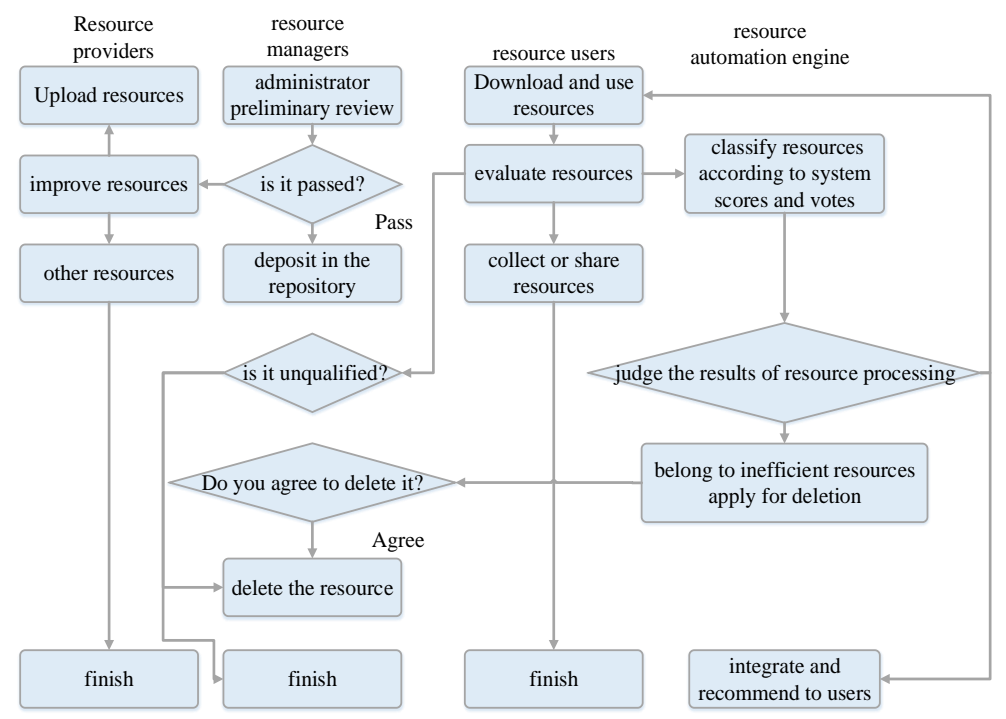

Fig. 9. Teaching resource management and optimization mode of Tai Ji course in the sophomore year

The general framework of Tai Ji course teaching system based on SNS network teaching is shown in Fig.10. The system is composed of three levels: presentation layer, business logic layer and data access layer. Each layer depends on the services provided by the next layer, and offers the packaged services to the last layer. Teachers and students interact with the system through the user interface provided by the presentation layer.

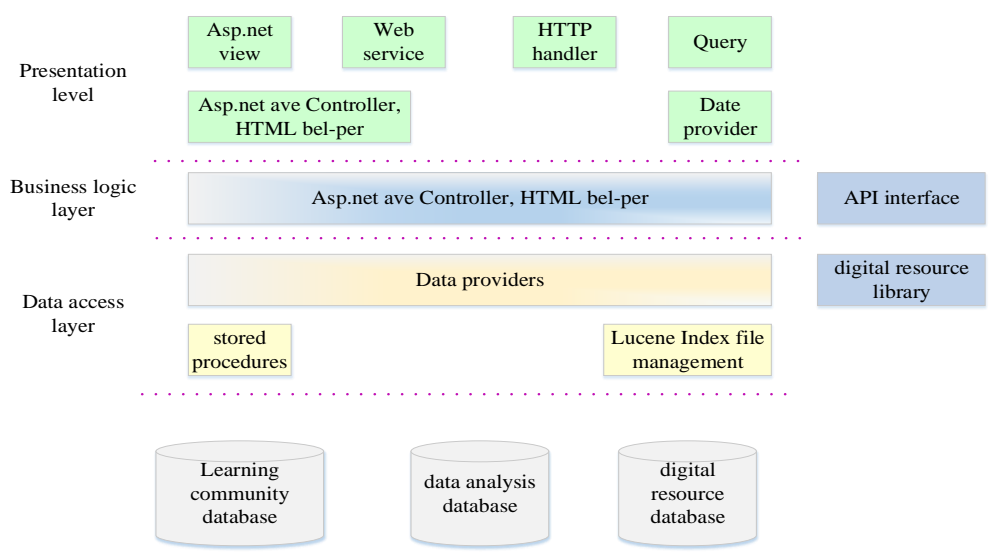

Fig. 10. General framework of Tai Ji course teaching system based on SNS network teaching

In this study, a kind of virtual software of Tai Ji teaching was used, called "TaijiZen Yun Shou". "Tai Ji Chan Yun Shou" is a teaching App which is researched 
and developed by TaijiZen International Culture Development Co., Ltd. Its iOS version displays 3D learning mode of TaijiZen Yun Shou through the form of 3D animation. The users can rotate the learning interface $360^{\circ}$ to view different angles of different movements. The application presentation is shown in Fig.12 and Fig.13. The software can apply virtual 3D technology and multi-functional operating interface to create a brand-new Tai Ji learning mode in the teaching process, and is built in English and Chinese learning commands. Its advantage is that students can view the movement, skill and routine of TaijiZen Yun Shou from various angles. Fig.13 shows the application of SNS multimedia teaching by PE Teachers.

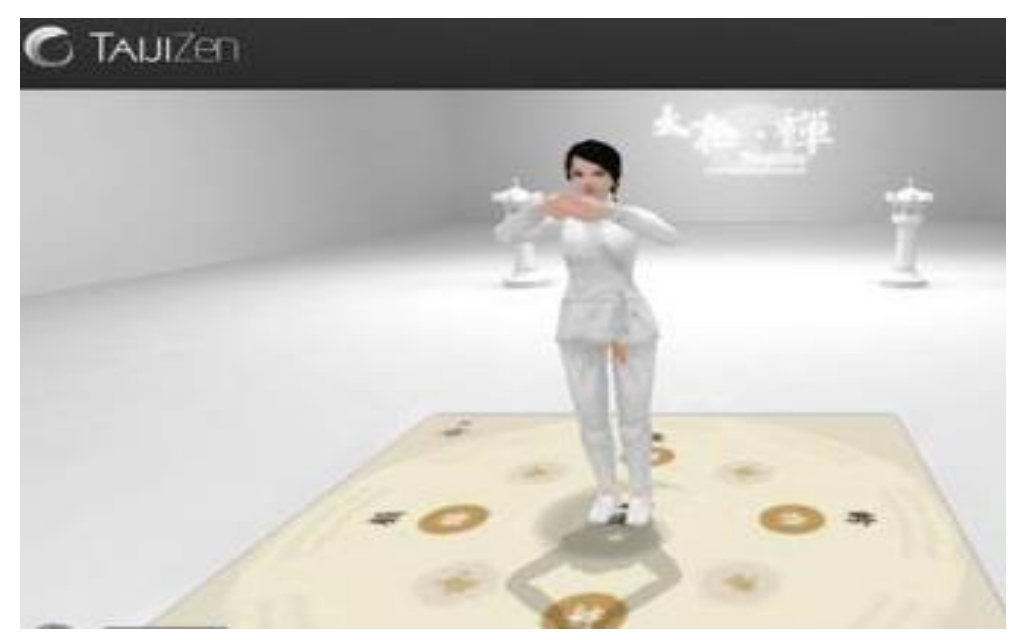

Fig. 11. Screenshot I of official version application of TaijiZen Yun Shou in SNS

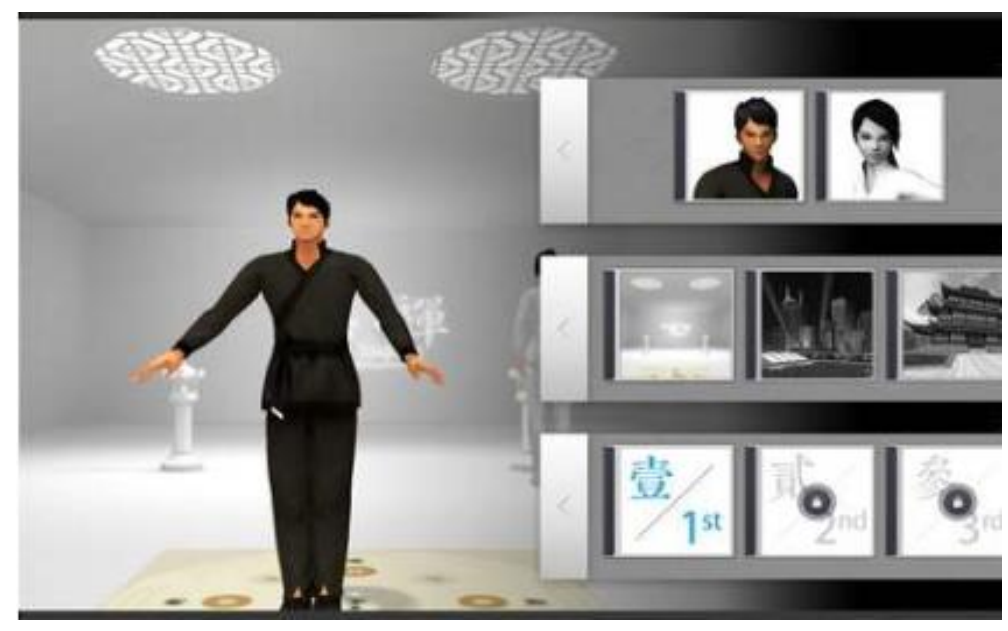

Fig. 12. Screenshot II of official version application of TaijiZen Yun Shou in SNS 


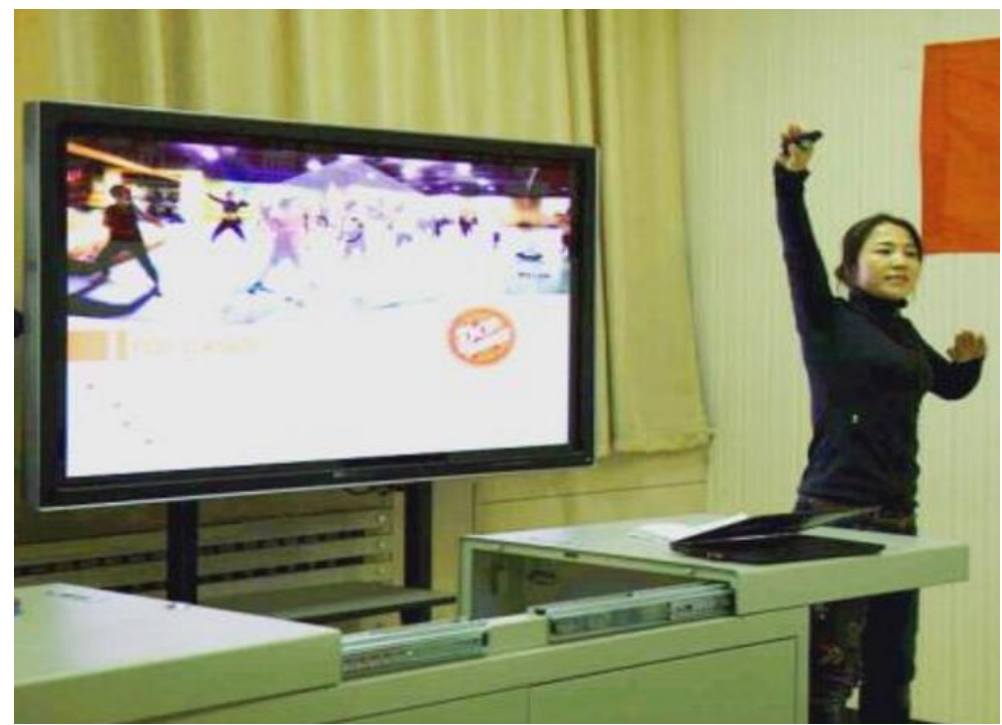

Fig. 13. Presentation of PE multimedia teaching application

\subsection{Teaching effect}

In order to test the teaching effect of SNS-based network teaching resource method, Tai Ji course in the sophomore year was taken for example for teaching practice. In the teaching practice, two horizontal classes were selected: control class (50 students) and experimental class (50 students). The traditional teaching mode was used for the control class, while SNS-based network teaching resource method was applied for the experimental class. After the completion of the teaching, the questionnaire was prepared to test the teaching effect of the two classes, and the distribution of test results is shown in Table 1. It can be seen from Table 1 that, the average score of experimental class is 90 , higher than the control class (84). In addition, the proportion of high scores (above 80) in the experimental class is also higher than that of control class, indicating that SNS-based network teaching resource method significantly improves students' scores in Tai Ji course.

Table 1. Teaching effect test results of both classes

\begin{tabular}{|c|c|c|c|c|}
\hline \multirow{2}{*}{ Score interval } & \multicolumn{2}{|c|}{ Experimental class } & \multicolumn{2}{c|}{ Control class } \\
\cline { 2 - 5 } & No. & Proportion & No. & Proportion \\
\hline$<60$ & 1 & $2 \%$ & 2 & $4 \%$ \\
\hline $60-70$ & 6 & $12 \%$ & 8 & $16 \%$ \\
\hline $70-80$ & 14 & $28 \%$ & 20 & $40 \%$ \\
\hline $80-90$ & 24 & $48 \%$ & 19 & $38 \%$ \\
\hline $90-100$ & 5 & $10 \%$ & 1 & $2 \%$ \\
\hline Average scores & \multicolumn{3}{|c|}{83.41} & \multicolumn{2}{c|}{75.72} \\
\hline
\end{tabular}




\section{Conclusion}

To solve the problem that there is lack of high-quality teaching resources in current PE teaching, a teaching mode based on SNS network teaching resource was designed in this study. The self-organization construction of teaching resources was achieved from building the PE teaching network learning community based on SNS to network courseware, teaching knowledge points, common problems, cases and media materials, etc. Meanwhile, for scientific and accurate evaluation of teachers' teaching ability, this study also proposed the teacher ability evaluation system applicable to SNS network teaching based on data mining method. Finally, the teaching design mode based on SNS network teaching was applied in teaching design and implementation of college Tai Ji course, and a kind of virtual software for Tai Ji teaching was also applied. Virtual 3D technology and multi-functional operating interface was used to create a brand-new Tai Ji learning mode in the teaching process. The results show that the average score of students for whom the new Tai Ji course teaching mode proposed in this study was used is higher than that of students receiving traditional teaching method, and the proportion of high scores is also higher. The teaching test effect demonstrates that this method can significantly promote students' scores. But, the teaching effect of the new teaching mode in this study still needs to be tested so as to promote and use it in more courses.

\section{Acknowledgement}

This work was supported by General Research Projects of Taizhou Polytechnic (2019JSJG630).

\section{$7 \quad$ References}

[1] Sullivan, S.L., Keating, X.D., Chen, L., Guan, J., Delzeit-McIntyre, L., \& Bridges, D. Physical Education and General Health Courses and Minority Community College Student Risk Levels for Poor Health and Leisure-Time Exercise Patterns. College Student Journal, 2008, vol. 42(1), pp. 132-151.

[2] Li, M.L., Ren, Y.J. A Multimedia Teaching Model for "Sports Statistics" Based on ARCS Motivation Theory. International Journal of Emerging Technologies in Learning , 2018, vol. 13(9), pp. 15-28. https://doi.org/10.3991/ijet.v13i09.8972

[3] An, D.Y. Analysis of the advantages of Taijiquan Teaching in Tianjin Medical University. The Guide of Science \& Education, 2015, vol. 12, pp. 113-113.

[4] Starcic, A.I., Barrow, M., Zajc, M., et al. Students' attitudes on social network sites and their actual use for career management competences and professional identity development. International Journal of Emerging Technologies in Learning, 2017, vol. 12(5), pp. 65-81. https://doi.org/10.3991/ijet.v12i05.6778

[5] Maier, C., Laumer, S., Weinert, C., \& Weitzel, T. The effects of technostress and switching stress on discontinued use of social networking services: a study of Facebook use. Information Systems Journal, 2015, vol. 25(3), pp. 275-308. https://doi.org/10.1111/ isj. 12068 
[6] Hsiao, K. Why internet users are willing to pay for social networking services. Online Information Review, 2011, vol. 35(5), pp. 770 - 788. https://doi.org/10.1108/14684521111 176499

[7] Lim, K. International students' use of social network services in the new culture: a case study with Korean youths in the United States. Asia Pacific Education Review, 2012, vol. 13(1), pp. 113-120. https://doi.org/10.1007/s12564-011-9173-x

[8] Hori, M., Ono, S., Kobayashi, S., et al. Learner Autonomy through the Adoption of Open Educational Resources Using Social Network Services and Multi-media E-textbooks. 2015, vol. 10(1), pp. 23-35. https://doi.org/10.1108/aaouj-10-01-2015-b004

[9] Moreno, M.A., Jelenchick, L.A., Egan, K.G., et al. Feeling Bad on Facebook: Depression disclosures by college students on a Social Networking Site. Depression \& Anxiety, 2011, vol. 28(6), pp. 447-455. https://doi.org/10.1002/da.20805

[10] Zheng, Y., Dong, J.D., Luo, H.Y., Zhang, X.L. Research on the Development of Teaching Resource-sharing Platform with Sns-oriented Ways in WLAN Environment. Software Engineer, 2014, vol. 16(2), pp. 35-37.

[11] Chen, H. Relationship between Motivation and Behavior of SNS User. JSW, 2012, vol. 7(6), pp. 1265-1272.

[12] Huang, B. Improving the management of colleges and Universities Based on the development of students and improving the teaching quality management ability of colleges and departments. China Economist, 2017, vol. 25(11), pp. 214-215.

[13] Zhao, W.K. The adaptive development of college physical education content. Science \& Technology of Stationery \& Sporting Goods, 2017, vol. 15(2), pp. 131-132.

\section{Authors}

Xuelin Yang is an associate professor in the Taizhou Polytechnic College, Taizhou, China (33923367@qq.com).

Xiaojun Jiang is an associate professor in the Taizhou Polytechnic College, Taizhou, China (33923367@qq.com).

Li Rong is a Researcher in the Taizhou Polytechnic College, Taizhou, China (33923367@qq.com).

Zhe Xu (Correspondence Author) is a Lecturer in the College of Information Science and Engineering, Hebei North University, China (124243157@ qq.com).

Article submitted 2020-01-23. Resubmitted 2020-02-28. Final acceptance 2020-02-30. Final version published as submitted by the authors. 